\title{
The Rise of Accounting Administration and Its Impact on the Profitability of Microeconomic Management
}

\section{La Administración Contable del Rise y Su Incidencia en la Rentabilidad de la Gestión Microeconómica}

VII International Congress of

Science, Technology,

Entrepreneurship and

Innovation (SECTEI 2020)

Corresponding Author:

E. Melendres Medina

emmelendres@hotmail.com

Published: 26 August 2021

Production and Hosting by

Knowledge E

(c) R. Acosta Velarde

et al. This article is distributed under the terms of the Creative Commons

Attribution License, which permits unrestricted use and redistribution provided that the original author and source are credited.
G OPEN ACCESS

\section{R. Acosta Velarde ${ }^{1}$, M. Campaña Lara ${ }^{2}$, E. Melendres Medina ${ }^{1}$, and J. Flores Dávila $^{1}$}

${ }^{1}$ Facultad de Administración de empresas, Escuela Superior Politécnica de Chimborazo, Extensión Morona Santiago, Riobamba, Ecuador

${ }^{2}$ Dirección de Investigación, Instituto Superior Tecnológico Stanford, Instituto Superior Tecnológico José Ortega y Gasset, Riobamba, Ecuador

\section{Abstract}

Some taxes may limit microeconomic growth. A greater growth of SMEs that use RISE as part of their administration, especially grocery stores, has been identified in the commercial sector in the city of Macas, Morona canton, Morona Santiago province, Ecuador. This has led to a lack of knowledge about their profitability because the nature of the tax can lead to reduced use of accounting tools. Therefore, the objective of this research was to analyze the accounting administration of RISE and its impact on the profitability of these businesses, and to propose the design of a tool that could contribute to the development of efficient management of the local microeconomic businesses. A deductive quantitative approach was used and data were collected through a survey and interviews. The Chi square test was used to analyze the results. According to the findings, the limitations faced by businesses that use this tax included the generation of monthly income that does not cover the monthly basic basket. Based on the results, we proposed a strategy for the efficient management of the microeconomy that is made up of four tools: conscious strategic planning, administration without tax barriers, efficient accounting tools, and the sustainable development of the company.

Keywords: administration, accounting, microeconomics, RISE, management, taxation.

\section{Resumen}

Algunos impuestos pueden limitar el crecimiento microeconómico, en la ciudad de Macas, cantón Morona, provincia de Morona Santiago, Ecuador se ha identificado un mayor crecimiento de PYMEs del sector comercial, de manera especial tiendas de abastos quienes utilizan el RISE como parte de su administración, lo que ha provocado un deficiente conocimiento real de su rentabilidad debido a la naturaleza misma del impuesto que lleva a un uso deficiente y casi nulo de los las herramientas contables, el trabajo tiene como objetivo analizar la administración contable del RISE y su incidencia en la rentabilidad de estos negocios que permitan el diseño de una herramienta que aporte al desarrollo de la gestión eficiente de la microeconomía local, se analizan datos obtenidos a partir de un estudio de tipo deductivo de enfoque cuantitativo, metodología de tipo analítico, correlacional, de campo que aplica instrumentos la recolección de información como la encuesta y entrevista, determina la dependencia de las variables identificadas a través del

How to cite this article: Velarde RA, Lara MC, Medina EM, Dávila JF (2021). The Rise of Accounting Administration and Its Impact on the Profitability of 
Chi cuadrado, e identifica como parte de los resultados las limitaciones a las que los negocios que utilizan este impuesto tienen como es la generación de ingresos mensuales que no llegan a cubrir la canasta básica mensual; plantea como parte de la solución al problema la Estrategia para la gestión eficiente de la Microeconomía que se compone cuatro herramientas: la planificación estratégica consciente, administración sin barreras tributarias, herramientas contables eficientes, el desarrollo sustentable de la empresa.

Palabras Clave: administración, contabilidad, microeconomía, rise, gestión, tributación.

\section{Introducción}

Las micro, pequeñas y medianas empresas son imprescindibles para el desarrollo económico de un país ya que uno de sus aportes es la generación de autoempleo y empleo para las localidades [1], siendo éstas las que existen en mayor número en Ecuador debe indicarse que la administración de una empresa o negocio debe llevarse en base a herramientas y pilares importantes como la Contabilidad, su aplicación va más allá de sus bases teóricas, dependerá de políticas estatales que delinean la contribución que el ejercicio económico de la empresa deberá aportar para el desarrollo. El sector comercio a diciembre 2019 representó el 17,9\% de participación dentro de la composición de los empleos por rama [2]. Como parte de este sector podemos citar las tiendas y abastos que constituye para la ciudad de Macas cantón Morona Santiago uno de los principales emprendimientos y negocios establecidos de la población urbana.

Ecuador a través del Servicio de Rentar Internas marca la ruta a seguir sobre las imposiciones tributarias a las que los empresarios y emprendedores deberán ajustarse en la tarea de la productividad, uno de estos impuestos y el más utilizado a nivel de la microeconomía es el Régimen Impositivo Simplificado (RISE), implementado en el país el 2007 para incorporar al sector informal en las contribuciones del Estado [3].

En la ciudad de Macas, provincia Morona Santiago del Ecuador, se identifica un gran incremento de PYMEs sobre todo del área comercial del sector urbano, donde se puede observar la generación de autoempleo a través de tiendas y abastos, estos establecimientos utilizan mayormente el RISE como parte de sus procesos de administración, lo que ha provocado un deficiente conocimiento real de su rentabilidad debido a la naturaleza misma del impuesto que lleva a un uso deficiente y casi nulo de los las herramientas contables incidiendo significativamente en la proyección del desarrollo microeconómico. 
Definida la problemática, se plantea como objetivos del estudio: el analizar la administración contable del Régimen Impositivo Simplificado (RISE) y su incidencia en la rentabilidad del negocio en la ciudad de Macas que permitan proponer una herramienta que aporte al desarrollo de la gestión microeconómica local.

\subsection{Tributos en Ecuador y las PYMEs}

Los sistemas tributarios permiten entre otros objetivos financiar la política y servicios públicos con una visión de lo social, para algunas regiones y países como España aunque registraron en algunos periodos un bajo nivel de recaudaciones fiscales, sus porcentajes fluctúan en virtud de la economía y el precio de bienes y/o servicios producidos, a la hora de medir la rentabilidad todo suma a nivel macroeconómico, Latinoamérica tiene ciertas similitudes al resto de las regiones con la imposición de gravámenes como lo es IVA o el impuesto directo conocido como Renta, estudios citados en el trabajo de Garzón, Ahmed y Peñaherrera [4] indican que el promedio del IVA mundial es del 15\% (dato al 2014), en Ecuador su valor es actualmente del $12 \%$ es una de las más bajas de nivel Latinoamericano.

El Sistema de Rentas Internas (SRI) recauda y controla los impuestos en el Ecuador, por otras instituciones nacionales, provinciales y municipales, con la finalidad de estimular la inversión, el ahorro, el empleo y la distribución de la riqueza nacional [5]. Los impuestos más importantes del país de acuerdo a la información estadística del SRI son los siguientes:

1. Impuesto a la Renta

2. Impuesto al Valor Agregado (IVA)

3. Impuesto a los Consumos Especiales (ICE)

4. Herencias, legados y donaciones

5. Salida de divisas

EI RISE reemplaza a los dos primeros impuestos detallados en líneas anteriores. Fue implementado en el Ecuador en el año 2008 mediante la Ley Reformatoria para la Equidad Tributaria LRET y ante la necesidad de contar con un sistema que regularice las transferencias de bienes y servicios de quienes poseen un negocio o local comercial y pago de sus impuestos que tenían un gran porcentaje de evasión, su objetivo es la exoneración de la declaración del impuesto al valor agregado (IVA) y del Impuesto a la Rente (IR) fijando una cuota para quienes no tengan ingresos superiores a $\$ 60.000$ anuales. De esta manera el presupuesto general de Estado estará alimentado entre otros por ingresos tributarios en los cuales el RISE forma parte [6]. 
Durante el año 2019 Ecuador recaudó por motivo del RISE \$21.903 y en 2018 \$20.435 logrando un incremento de del 6,7\% al año anterior, y aunque el impacto en dólares no pareciera ser significativa, se ha conseguido una mayor formalización en el sector comercio. El cantón Morona en el año 2019 logró una recaudación de impuestos tributarios de $\$ 9.781 .197$ [7].

Beneficios: EI RISE ha permitido facilitar el cumplimiento de obligaciones tributarias de los segmentos informales de comercio en el país. Siendo este un sector cada vez más creciente, es así que en 2018 en subempleo llegó a

878.428 personas a nivel nacional y para 2019 la cifra fue 914.201 personas. [8].

Quienes se acojan al RISE no necesitan usar formularios y el pago se realizará directamente en cualquier entidad del sistema financiero.

1. Al estar libres de retenciones, las personas inscritas en el RISE evitarán pagos en exceso.

2. Los inscritos en el RISE ahorrarán tiempo y dinero, pues no deberán contratar un contador que les lleve sus cuentas.

3. Los contribuyentes inscritos en el RISE entregarán notas de venta, así ahorrarán tiempo al llenarlas y no tendrán dificultades al hacerlo.

4. Por cada nuevo trabajador bajo contrato vigente que se encuentre afiliado al IESS, siempre que esté al día en sus pagos, obtendrá un descuento del 5\% en su cuota.

Pueden inscribirse los pequeños negocios con ventas anuales inferiores a $\$ 60.000$ y con menos de 10 empleados; pequeños negocios cuyos propietarios, además, perciban ingresos anuales en relación de dependencia, menores a la fracción básica del Impuesto a la Renta y, que en conjunto con las ventas del negocio, no superen los $\$ 60.000$ anuales.

Actividades que pueden inscribirse

1. Agricultura

2. Pesca

3. Ganadería

4. Avicultura

5. Minería

6. Transporte

7. Manufactura

8. Servicios de restaurantes y hoteles 
9. Servicios de construcción

10. Trabajo autónomo

11. Comercio minorista

12. Otros servicios en general

Responsabilidades del contribuyente

1. Actualizar la información cuando supere o reduzca sus ingresos registrados al momento de inscribirse en el RISE.

2. Pagar su cuota mensual puntualmente o pre pagar el año.

3. Emitir comprobantes de venta autorizados, exclusivamente, por las transacciones propias del contribuyente.

La cuota mensual a cancelar por parte del contribuyente se establece en función de 7 categorías:

\section{Table 1}

Montos máximos en comprobantes de venta RISE.

\begin{tabular}{l|ll} 
Categorías & Monto de ingresos & Cuota RISE \\
1 & 0 a 5.000 & 420,00 \\
\hline 2 & De 5.001 a 10.000 & 850,00 \\
\hline 3 & De 10.001 a 20.000 & $1.700,00$ \\
\hline 4 & De 20.001 a 30.000 & $2.500,00$ \\
\hline 5 & De 30.001 a 40.000 & $3.350,00$ \\
6 & De 40.001 a 50.000 & $4.200,00$ \\
7 & De 50.001 a 50.000 & $5.000,00$
\end{tabular}

Fuente: Basado en SRI www.sri.gob.ec/web/guest/regimen-impositivo-simplificado-rise

\section{Base legal}

1. Constitución de la República del Ecuador. Artículos: 66 y 300.

2. Ley de Creación del Servicio de Rentas Internas. Artículos: 2 y 9.

3. Código Tributario. Artículos: 115, 116, 119, 120, 121, 124, 125, 126, 127, 128, 129, 130, $131,132,133,134$ y otros.

4. Ley de Régimen Tributario Interno. Artículos: 97,1 y siguientes.

5. Reglamento para la aplicación de la Ley de Régimen Tributario Interno. Artículos: 215 y siguientes. 
6. Resolución No. NAC-DGERCGC16-00000155.

7. Resolución No. NAC-DGECCGC10-00013.

PYMEs: Es un tipo de clasificación de empresas, cuya diferenciación radica en su tamaño. Loor et al. [9] señalan que son Pequeñas y medianas empresas, cuya organización jurídica destaca que el 97\% posee Registro Único de Contribuyente.

Registro administrativo: 'Es todo registro resultante de necesidades fiscales, tributarias u otras, creado con la finalidad de viabilizar la administración de los programas de gobierno o para fiscalizar el cumplimento de obligaciones legales de la sociedad' [10] (p. 10)

En el Ecuador las empresas a se clasifican según su tamaño, tomando en cuenta los ingresos sobre el número de trabajadores de la siguiente manera:
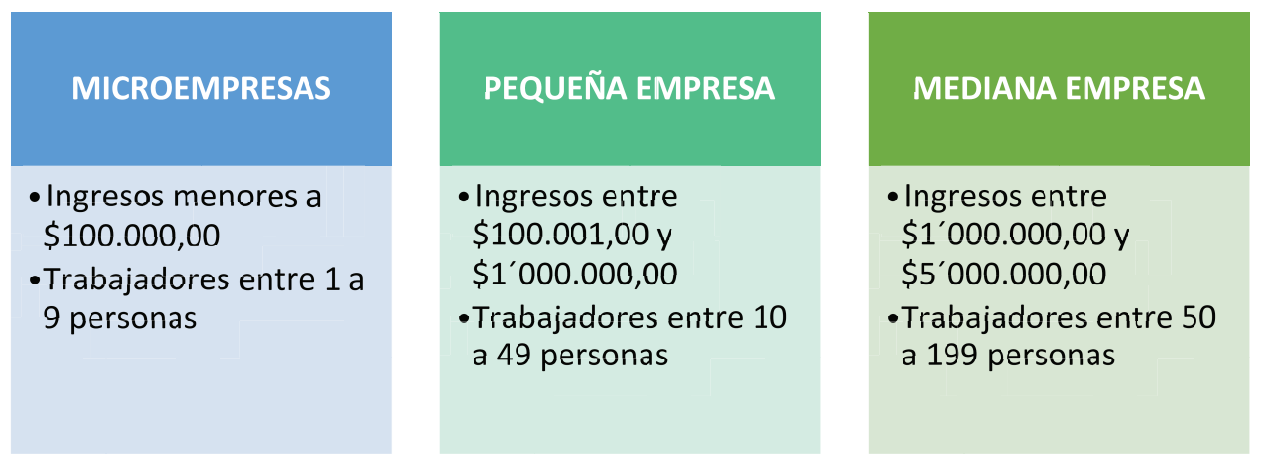

Figure 1

Tipo de empresas. Fuente: Basado en Código Orgánico de la Producción, Comercio e Inversiones (2017).

Las micro empresas y pequeñas empresas son motivo de este estudio, la mayoría de éstas se desarrollan en el área del comercio, en Ecuador el 51,16\% de las empresas se dedican al comercio, permitiendo una ocupación del 66,17\% de la mano de obra a nivel nacional. El total de personal ocupado en las grandes y medianas empresas es de 1 '000.213 personas de las cuales el $69,42 \%$ son hombres y $30,58 \%$ mujeres $(694.411$ son hombre y 305.802 mujeres), los trabajadores de los servicios y vendedores de comercios y mercados representa el 25,49\% según datos del INEC [11], ocupando el segundo lugar de aportes a nivel nacional con $\$ 11.721$ millones de dólares con productos como el azúcar, harina, arroz, leche pasteurizada que son los de mayor producción y expendio en los pequeños negocios, las producción de pan también logró uno de los puestos más importantes en le 2016, este grupo de empresas se las conoce también como PYMEs.

Su contribución a la economía local como podemos observar está en la generación de empleo, participan el $50 \%$ de la producción y crean casi el $100 \%$ de los servicios que 
usa la población, permiten el desempeño económico de la clase media hacia abajo en el PIB [12]

Conformación del directorio de empresas y establecimientos, de acuerdo al INEC 2016 determina el número de empresas que se encuentra en el país. El DIEE (2016) señala las siguiente:

1. Existen 843.745 empresas en el año 2016 que comprenden unidades económicas que registraron alguna de las siguientes condiciones: (1) Ventas en el SRI, (2) Personal afiliado en el IESS, (3) Perteneciendo al RISE pagaron impuestos sobre sus ingresos en el SRI.

2. De este número de empresas, 368.651, es decir, el 43,7\%, registran información únicamente de personal afiliado, esta situación se da principalmente en microempresas y pequeñas empresas cuyos ingresos no superan la base imponible y por tanto no están obligadas a declarar el Impuesto a la Renta en el SRI.

3. 362.854 empresas que representan el $43,0 \%$ son empresas que declararon como RISE, cuyos ingresos por ventas anuales son menores a 60.000 dólares y no registran personal afiliado.

4. 21.934 empresas que representan el $2,6 \%$, son empresas que presentan solo información de ventas, esta situación se da principalmente en microempresas y pequeñas empresas unipersonales o familiares cuyo flujo de ingresos es irregular como en los sectores de la construcción, comercio minorista e inmobiliarias y que por su naturaleza no necesariamente registran el personal ocupado en el IESS.

5. 4.066 empresas que representan el 0,5\%, cuentan con información de ventas y personal afiliado de las actividades $\mathrm{O}, \mathrm{P}$ y $\mathrm{Q}$.

6. 86.240 empresas que representan el 10,2\%, cuentan con información de ventas y personal afiliado de actividades productivas, se excluye las actividades O, P y Q.

De acuerdo al ejercicio económico 2019 de la Superintendencia de Compañías, Valores y Seguros, las microempresas se ubican en el primer lugar del ranquin empresarial según el tamaño con un aporte del 51,3\% del PIB [13].

\subsection{Rentabilidad en los negocios}

El término rentabilidad se identifica como el beneficio obtenido luego de realizar la actividad comercial en un periodo de tiempo. Pérez [14] se refiere a los beneficios conseguidos o que pueden obtenerse procedentes de una inversión realizada con anterioridad. Este concepto resulta muy importante tanto en el ámbito empresarial como en el de las inversiones, ya que permite conocer la capacidad de una compañía 


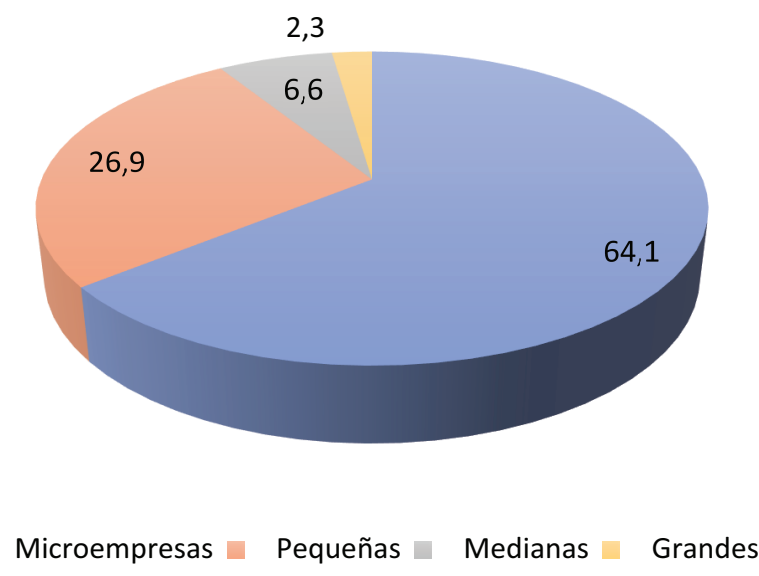

Figure 2

Ranking de compañías de acuerdo a su tamaño. Fuente: Referencia [13].

para remunerar los recursos financieros empleados. Hay que distinguir básicamente entre dos clases de rentabilidad, como son la rentabilidad financiera y rentabilidad económica:

1. Rentabilidad financiera: Este concepto de rentabilidad financiera engloba el beneficio que consiguen los distintos socios de un negocio. Se encarga de medir la capacidad de una empresa para generar ingresos a partir de sus fondos. Hace referencia a la relación entre el beneficio neto y el patrimonio neto de la compañía. La rentabilidad financiera se puede mejorar de varias maneras: Incrementando la deuda para que resulte mayor la diferencia entre el activo y los fondos propios, Incrementando el margen, Reduciendo el activo o aumentando las ventas.

2. Rentabilidad económica: Es un tipo de rentabilidad expresada en porcentaje. Cantero and Leyva [15] expresa que es un medio que tienen las compañías debido a las distintas inversiones realizadas. Si en un ejercicio se obtiene una rentabilidad de un $20 \%$ implica que por cada 100 euros invertidos ha obtenido de ganancias 20. Es un indicador de eficiencia que evalúa los resultados de una empresa. Se ocupa de comparar el resultado conseguido con el desarrollo de la actividad de la compañía con las inversiones hechas. Ese resultado aún será menor al tener que restarle impuestos, gastos e intereses.

Cada empresa deberá adoptar los métodos más adecuados para su negocio con el ánimo de incrementar la rentabilidad económica. La manera más sencilla y utilizada pasa por rebajar los costes empresariales ordinarios y subir el precio de los productos o bienes.

La rentabilidad económica se consigue sobre todo de dos aspectos, el margen de beneficios y el número de ventas, por lo que si no somos capaces de elevar el margen, la solución que queda pasará por mejorar las ventas. 
La fórmula para calcular la rentabilidad económica de una empresa es la siguiente: BAll: Beneficio antes de Intereses e Impuestos o Beneficio Bruto; es el beneficio que obtiene la empresa antes de descontar los gastos financieros, intereses e impuestos que tiene que pagar por desarrollar su actividad económica.

La administración de una empresa o negocio debe llevarse en base a herramientas y pilares importantes como la Contabilidad. Mejía [16]define la Contabilidad como una ciencia social aplicada que permite una valoración cuali y cuantitativa de la riqueza controlada por las empresas, definición referida en el artículo científico Fundamentación Teórica de los modelos en Contabilidad publicada en la Revista Científica Estudiantil Innovación contable. Su aplicación va más allá de sus bases teóricas, dependerá de políticas estatales que delinean la contribución que el ejercicio económico de la empresa deberá aportar para el desarrollo social.

\section{Materiales y Métodos}

La investigación aplicada tiene un enfoque cuantitativo determinando variables como: La administración contable del RISE y su incidencia en la rentabilidad de las tiendas y comercios midiendo el fenómeno a partir de una muestra aleatoria de la ciudad de Macas, utiliza métodos inductivos y deductivos para la obtención de información y estudios bibliográficos, de campo, analíticos y correlacionales con técnicas como la encuesta y la observación apoyados en instrumentos como cuestionarios y fichas, que permitan el análisis de los hechos y el diseño de herramientas de gestión que permitan optimizar la microeconomía. Se estudian para ello cuestionamientos teóricos, económicos, tecnológicos, sociales, estadísticos que se presentan en este estudio.

El desarrollo de cada una de las variables se lo realiza con las siguientes áreas:

La administración contable del RISE y su incidencia en la rentabilidad de los negocios:

1. Conocimiento de obligaciones tributarias a cumplir con el SRI

2. Cumplimiento de obligaciones tributarias

3. Ventajas y desventajas de la utilización del RISE

La rentabilidad de las tiendas y comercios

1. Tiempo de funcionamiento del negocio

2. Ingreso mensual

3. Gastos mensuales

4. Inversión inicial

5. Expectativas de ampliación 
6. Identificación de ganancia del ejercicio del negocio

7. Control de inventarios o mercadería

8. Tipo de instrumento para registro y control de las operaciones del negocio

9. Rentabilidad

\subsection{Población y muestra}

Según el SRI, IESS, Ministerio de Educación, Superintendencia de Bancos y Encuestas Internas del INEC las Empresas y establecimientos a nivel nacional registrados en el SRI en el 2016 a nivel nacional es de 843.745 empresas y 978.490 establecimientos, datos con una cobertura geográfica provincial, cantonal y parroquia rural, de donde la micro empresa a nivel nacional representa el 90,51\%, (763.636 establecimientos), la provincia de Morona Santiago congrega una participación en ventas según provincia, año 2016 del 0,1\%.

Cálculo de la muestra:

$$
\begin{gathered}
n=\frac{Z^{2}(p)(q)(N)}{N(e)^{2}+(Z)^{2}(p)(q)} \\
n=\frac{(1,96)^{2}(0,5)(0,5)(764)}{764(0,05)^{2}+(1,96)^{2}(0,5)(0,5)} \\
N=256 .
\end{gathered}
$$

Se realizan por tanto 256 encuestas tomando como grupo aleatorio de las tiendas de abastos y comercios pequeños de la ciudad de Macas, provincia de Morona Santiago, Ecuador.

\section{Resultados y Discusión}

En una primera etapa se identifica y describe la administración contable del RISE en los negocios, de donde solo $48,8 \%$ de encuestado ha considerado el RISE como parte de su cumplimiento tributario, de acuerdo a la Tabla 2.

Del total de la población solo el $16 \%$ considera que el incorporar a su administración contable el RISE tenga ventajas, y del $49 \%$ que han incorporado el RISE como herramienta tributaria el $61 \%$ indica que no encuentra ventajas para su negocio como se puede observa en la Figura 4.

Entre las principales razones que mencionan los encuestados están: La limitación en la generación de ingresos puesto que al superar las bases se pierden beneficios, 
Table 2

Determinación de la administración contable de impuestos.

\begin{tabular}{l|ll}
$\begin{array}{l}\text { Administración contable de } \\
\text { impuestos }\end{array}$ & Frecuencia & Porcentaje \\
\hline RISE & 125 & 48,8 \\
RUC & 124 & 48,4 \\
\hline Ninguno & 7 & 2,7 \\
\hline Total & 256 & 100,0
\end{tabular}

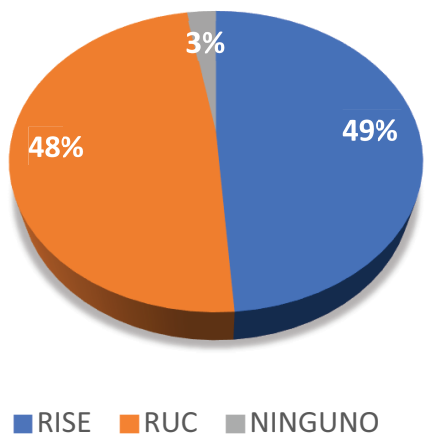

Figure 3

Determinación de la administración contable de impuestos.

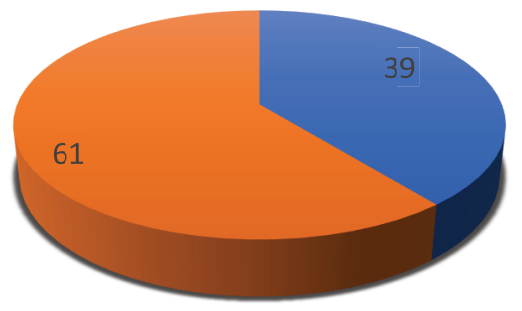

Si $n$ No

Figure 4

Determinación de la administración contable de impuestos.

un menor control de los ingresos reales para el negocio, menor uso de herramientas contables.

La administración contable de impuestos está dividida en un 50\% aproximadamente entre el Régimen impositivo simplificado RISE y el Registro único de contribuyentes o RUC, su eficiencia en la rentabilidad se estudia en la siguiente tabla, considerando que los rangos con los cuales se diseña la presentación de los datos obtenidos se encuentran por debajo del salario básico mensual. 
Table 3

Rentabilidad anual de los negocios de la cuidad de Macas.

Rentabilidad anual
$0-1000$
$1001-5000$
$5001-$ mas
Total

Frecuencia
99
73
84
256

Porcentaje
38,7
28,5
32,8
100,0

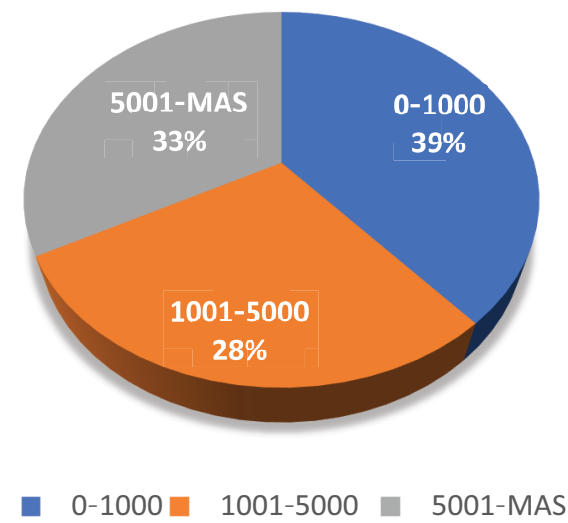

Figure 5

Porcentaje de Rentabilidad anual de los negocios de la cuidad de Macas

La rentabilidad de manera general es baja en los negocios de la ciudad de Macas, el $67 \%$ de negocios obtienen una rentabilidad inferior a la canasta básica actual (aprox $\$ 400$ mensuales), como se describe en Figuras 5 y 6.

\section{Table 4}

Rentabilidad anual de los negocios de la cuidad de Macas y su relación con los impuestos aplicados.

\begin{tabular}{l|l|l|l|l} 
Impuestos & $\mathbf{0 - 1 0 0 0}$ & $\mathbf{1 0 0 1 - 5 0 0 0}$ & $\mathbf{5 0 0 1 - m a s}$ & Total \\
\hline RISE & 51 & 49 & $\mathbf{2 5}$ & $\mathbf{1 2 5}$ \\
RUC & 47 & 21 & 56 & 124 \\
Ninguno & 1 & 3 & 3 & 7 \\
Total & 99 & 73 & 84 & 256
\end{tabular}

De acuerdo a la gráfica que precede, la mayor rentabilidad anual la tienen los negocios que trabajan con el RUC, lo que corresponde al 56\% de la población en estudio. A simple observación se puede establecer que el RISE limita la aplicación de herramientas administrativas para una mayor rentabilidad.

La relación de dependencia entre las dos variables se analiza enseguida.

La comprobación de la Hipótesis se realiza por el método estadístico Chi- cuadrado $\left(x^{2}\right)$. La prueba resultante puede ser aplicada para variables tanto cualitativas como 


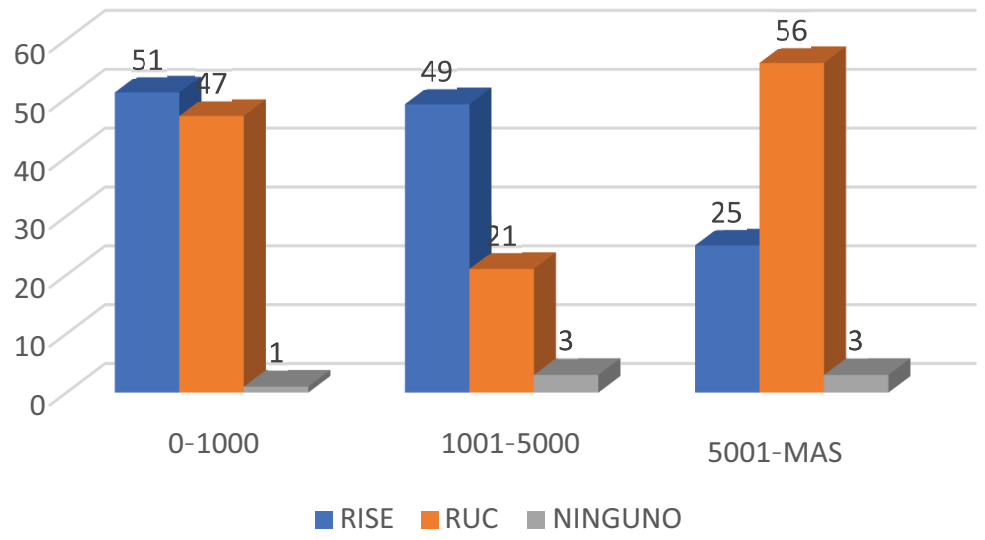

\section{Figure 6}

Análisis de la rentabilidad anual de los negocios de la cuidad de Macas y su relación con los impuestos aplicados.

cuantitativas, estableciendo la dependencia que existe en: La administración contable del RISE y su incidencia en la rentabilidad de los negocios de la ciudad de Macas, las dos variables en estudio.

Para el análisis las frecuencias observadas se analiza la presencia de la administración contable del RISE y el RUC, para el caso de la rentabilidad se establecen tres rangos de ingresos anuales en dólares: de 0 a 1.000, 1001-5000 y 5001 a más midiendo cuantitativamente la existencia de parámetros que determinan la dependencia existente entre administración contable del RISE entre los negocios que aplican este impuesto, así se obtiene la Tabla 5 .

\section{Table 5}

Frecuencias observadas de las variables.

\begin{tabular}{|c|c|c|c|c|c|c|c|}
\hline \multirow[t]{2}{*}{ Impuestos } & \multicolumn{7}{|c|}{ Rentabilidad anual } \\
\hline & $0-1000$ & & $1001-5000$ & & 5001-MAS & & TOTAL \\
\hline RISE & 51 & 48,34 & 49 & 35,64 & 25 & 41,02 & 125 \\
\hline RUC & 47 & 47,95 & 21 & 35,36 & 56 & 40,69 & 124 \\
\hline Ninguno & 1 & 2,71 & 3 & 2,00 & 3 & 2,30 & 7 \\
\hline Total & 99 & & 73 & & 84 & & 256 \\
\hline
\end{tabular}

Comprobación de hipótesis: 
...

La administración contable del RISE es independiente de la rentabilidad

$\mathbf{X}^{2} \mathbf{a}$

La administración contable del RISE no es independiente de la rentabilidad

$\mathbf{X}^{2}$ 。

$$
\begin{aligned}
& =\sum_{i=1}^{k}\left(\frac{(O i-E i)(O i-E i)}{E i}\right) \\
& =\sum_{i=1}^{k}\left(\frac{(99-51)(99-51)}{4}\right)
\end{aligned}
$$

$X^{2}{ }^{2}=24,8$

Valor crítico de $a=0,05$

$\mathbf{X}^{2}=(r-1)(c-1)$

$\mathrm{X}^{2}{ }_{\mathrm{a}}=7,8$.

En base a los resultados obtenidos se determina que la administración contable del RISE y la rentabilidad son dependientes.

Si bien la tributación se puede comprender a través del compromiso social, el redireccionamiento hacia el servicio común debe considerar las particularidades de los sectores productivos para promover su desarrollo haciéndolos equitativos para prevenir su desinterés, la evasión tributaria y minimizar los riesgos y perjuicios para las empresas y todo el sistema económico, es imprescindible lograr un equilibrio entre la redistribución y la eficiencia que permita el cambio de la matriz productiva [17].

A partir del estudio podemos determinar que si bien el pago de impuestos al SRI y recaudaciones de negocios pequeños o PYMEs creció para el Estado a partir de la implementación del RISE, para el emprendedor se ha convertido en una limitante de crecimiento ante los temores de la pérdida de ciertos beneficios como un pago menor de impuesto al mantener un margen de ganancia bajo que no sobrepase las bases imponibles, por otra parte, el facilitar la declaratoria fija de una cuota a través del RISE hace que los emprendedores no lleven sistemas simples contables que ocasiona una administración deficiente de sus negocios. Por lo general quienes emprenden estos negocios pequeños lo hacen por necesidades de autoempleo, bajos niveles de capital humano, dificultades para acceder a financiamientos externos o los escasos medios tecnológicos para el desempeño comercial, si bien del otro lado están pymes mas exitosas lo hacen gracias a una administración más eficiente e innovadora [18].

Las barreras tributarias medidas por el número de pagos por año en el caso de Ecuador llega a $664 \mathrm{hr}$ si hablamos de los tres impuestos más importantes lo cual es 
un valor demasiado alto, sumado a las dificultades en el reclamo de reembolsos en los cuales los contribuyentes no quieren verse envueltos vuelve a los sistemas tributarios un gasto más elevado para las PYMEs, la política actual no cuenta con estrategias direccionadas hacia este sector en particular, y a pesar de que el país tenga un gran componente de estas empresas no redirecciona sus acciones hacia el desarrollo de las mismas lo que dificulta el cambio de la matriz productiva y se subestima a este sector de la economía llevándolos a la informalidad, aunque su representación sea del 75\% a 65\% (microempresas), siendo el espacio que se ha creado la población como una oportunidad de autoempleo al no encontrar fuentes de trabajo en la pocas grandes empresas existentes [19].

El análisis de los comportamientos locales hace que las políticas relacionadas con empresas no puedan tener recetas comunes, y promuevan el aumento de la productividad, la innovación y motiven al emprendedor a crecer, el desarrollo económico debe provocar una mayor rentabilidad individual de las empresas en todas sus formas lo que conlleve a un crecimiento colectivo [20]. Es lo que llamaremos una microeconomía eficiente, planteando para su desempeño cuatro estrategias a partir de las observaciones realizadas del estudio de campo.

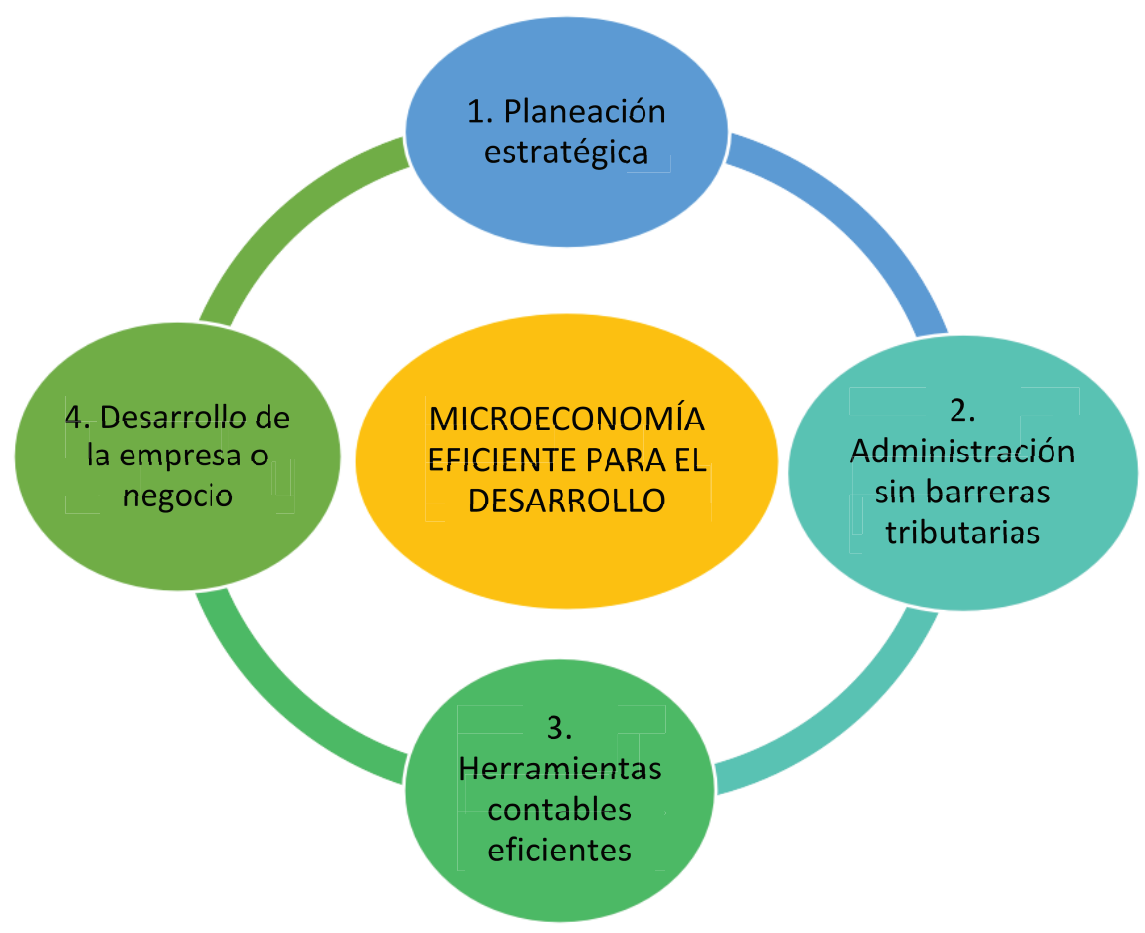

Figure 7

Estrategia para gestión eficiente de la Microeconomía.

La Microeconomía puede lograr ser más eficiente si se combinan elementos que permiten una gestión más ágil sin barreras de crecimiento. La estrategia para la gestión 
eficiente de la Microeconomía se compone de 4 elementos como se muestra en la Figura 7:

1. Planeación estratégica consciente: Una de las herramientas fundamentales para mantener una gestión eficiente es la Planificación. Pérez y García (17 de mayo de 2016) [21]señala 'Planificar es un proceso constante que involucra una serie de elementos a considerar, tales como: el tiempo disponible, los recursos, los propios intereses, los macro objetivos o grandes, metas en cada semestre y en la carrera en sí' (p. 20). El conocimiento de recursos y sectores productivos locales puede proyectar mejores resultados al construirse recetas únicas de acuerdo a las particularidades existentes [22].

Una proyección al futuro de la empresa es importante para su crecimiento, en base a la Planificación Estratégica consciente se analiza, actuales circunstancias desde diferentes entornos, lo que permite la toma de decisiones más acertada.

2. Administración sin barreras tributarias: Es inherente de la administración una concepción integral, donde la gestión tributaria que llevará a cabo la empresa debe ir acorde con su planificación permitiéndole proyectarse y romper barreras, con menores tiempos invertidos en las declaraciones de impuestos y con consideraciones acordes a la naturaleza misma de su existencia [23].

Una deficiente administración tributaria limita el crecimiento de la empresa, pues el temor de un mayor pago al Estado cuarta la búsqueda de mejores y mayores ingresos.

3. Herramientas contables eficientes: Saber el movimiento contable, aplicar herramientas para el registro, control y evaluación de ingresos y gastos es una alternativa que permitiría disminuir el temor a crecer empresarialmente, dotando a la empresa bases de una operación más exitosa.

Previo al establecimiento de la rentabilidad se debe considerar el cálculo de impuestos a pagar. Los impuestos afectan la rentabilidad de las empresas en el sentido de que obtienen un menor margen de rentabilidad restringiendo su crecimiento y desarrollo, recurriendo a medidas tan impactantes para su comercio como el aumento del precio de venta final afectando su competitividad llevando finalmente al cierre de sus actividades. Esto además, genera el aumento de la evasión fiscal la cual se ve por los empresarios como una consecuencia lógica y justificada, provocando la competencia desleal por parte de negocios informales que no cumplen las condiciones legales y por tanto generando inequidad en el sentido de que las empresas formales cumplen con su carga obteniendo menores ganancias, en cambio los informales no cumplen con la carga tributaria obteniendo mejores ganancias porque no tienen necesidad de repercutir los impuestos en el precio al consumidor. Al final, se resume en una frase expuesta por el autor: 'quien 
va a poder crear una empresa si en el primer año, ya tiene que pagar más de lo que ha ganado'.

4. Desarrollo sustentable de la empresa o negocio: El desarrollo involucra un equilibrio entre lo social, económico y ambiental, si la empresa no se proyecta al futuro y toma en cuenta herramientas administrativas, la innovación, proyección, etc., su economía será deficiente. El autoempleo generado no verá un incremento de sus ganancias, las pequeñas empresas deben implementar su maquinaria y equipo, con apoyo de los gobiernos a través del acceso a financiamiento para permanecer en el tiempo [24].

\section{Conclusiones}

La administración contable en los negocios y tiendas de la ciudad de Macas se distribuye en un $50 \%$ de la población estudiada que se ha decidido por el Régimen Impositivo Simplificado (RISE) sin embargo registran los niveles más bajos de rentabilidad puesto que el mayor rango, mayor a $\$ 5000$ anuales supera en un $50 \%$ a los negocios que se administran a través del RUC por tanto la incidencia en la rentabilidad es deficiente lo que hace más complicada la gestión de la microeconomía, el crecimiento individual no se genera lo que afecta a lo global ya que la administración contable del RISE y la rentabilidad son dependientes.

Contar con el RISE como medio impositivo del pago de impuestos influye significativamente en el desarrollo de los negocios y tiendas de la cuidad de Macas, ya que limita el nivel de rentabilidad que estas pueden tener, manteniendo sus niveles de ingresos por debajo de $\$ 5000$.00 anuales, lo que equivale a ingresos por motivo de la actividad comercial inferiores a la actual canasta básica demostrando su ineficiencia para el desarrollo empresarial aunque para el Estado representa ingreso para el quehacer público.

Este particular se agudiza ante el temor del empresario por tener que pagar más impuestos si crece, por tanto prefiere mantenerse bajo este régimen, a esto se suma el desconocimiento de herramientas contables que les permitan proyectarse para una gestión eficiente. El comportamiento de la rentabilidad en los negocios que están en el marco del RISE, es limitado, observándose un mayor número de micro empresas que no superan los $\$ 5000.00$ no así quienes optaron por el RUC, que logran tener una rentabilidad más eficiente que los proyecta a mejorar aún más su competitividad en el mercado. En base a estos los resultados se diseña la Estrategia para la gestión eficiente de la Microeconomía que se compone de 4 elementos: (i) La planificación estratégica, el diseño de; (ii) Administración sin barreras tributarias; (iii) Herramientas contables eficientes basadas en los diagnósticos proporcionados por el primer componente; (iv) 
Desarrollo de la empresa o negocio, basado en un equilibrio individual que promueva el grupal, i que impulsa una gestión eficiente de la micro economía.

\section{References}

[1] Valenzuela I, Valenzuela B, Irarrazaval J. Desarrollo emprendedor latinoamericano y sus determinantes: Evidencias y desafíos. Pilquen. 2018;21:55-63.

[2] INEC Ecuador. ENEMDU. Quito: Intrituo Nacional de estadísticas y Censos Ecuador; 2019.

[3] Endara M. RISE 10 años de vigencia, evolución, desafíos y perspectivas. Quito: Trabajo de graduación para maestría de la Universidad Andina Simón Bolívar; 2018.

[4] Garzón M, Ahmed A, Peñaherrera J. El sistema tributario y su impacto en la Economía Popular y Solidaria en el Ecuador. Uniandes Episteme. 2018;51:38- 53.

[5] Constitución de la República del Ecuador. Montecristi: Asamblea Nacional del Ecuador.

[6] Ruso J, Villamar D, Ordeñana A, Contreras E. Análisis de la recaudación del régimen impositivo simplificado ecuatoriano (RISE) y su importancia en los ingresos tradicionales del Presupuesto General del Estado. Periodo 2008-2017. INNOVA Research Journal. 2019;4:53-64.

[7] Sistema de Rentas Internas (SRI). Recaudación de gestión del servicio de Rentas internas Enero - Diciembre 2019. Quito: SRI; 2019.

[8] Instituto Nacional de Estadísticas y Censos (INEC). Encuesta Nacional de Empleo, Desempleo y Subempleo (EMENDU). Quito: INEC; 2019.

[9] Loor H, Ureta D, Rodríguez G, Cano E. Análisis del contexto socio- económico, comercial, financiero e internacional de las PYMEs Ecuatorianas. ECOCIENCIA. 2018; 6.

[10] CEPAL. Registros Administrativos calidad de los datos y credibilidad pública, presentación y debate de los temas sustantivosde la segunda reunión de la Conferencia Estadística de las Américasde la CEPAL. Serie Estudios Estadísticos y Prospectivos. 2017; 10.

[11] INEC. Encuesta estructural empresarial, una mirada a la producción en el Ecuador. Quito: INEC; 2016.

[12] Ron A, Sacoto V. Las PYMES ecuatorianas: su impacto en el empleo como contribución del PIB PYMES al PIB total. Espacios. 2017;38:15.

[13] Superintendencia de Compañías, Valores y Seguros. Ranking empresarial 2020. Quito: Superintendencia de Compañías, Valores y Seguros; 2019.

[14] Pérez R. Modelo de modernización para la gestión de organizaciones. EAN. 2020;46. 
[15] Cantero CH, Leyva C. La rentabilidad económica, un factor para alcanzar la eficiencia empresarial. Ciencias Holguín; 2016.

[16] Soto EM. Estructura General de la Teoría pura, los modelos y sistemas en Contabilidad. Colombia: Encuentro de la Red de Filosofía, Teoría y Educación en Administración; 2011.

[17] Segura S, Segura E. Las recaudaciones tributarias y el crecimeinto económico. Un análisis a través del PIB de Ecuador. Revista Empresarial. 2017;11:34-40.

[18] CEPAL, Euro mipyme. MIPYMES en América Latina Un frágil desempeño y nuevos desafíos para las políticas de fomento. Santiago: Naciones Unidas; 2018.

[19] OECD, Banco de Desarrollo de América Latina (CAF). América Latina y el Caribe 2019: Políticas para PYMES competitivas en la Alianza del Pacífico y países participantes de América del Sur. Paris: OECD; 2019.

[20] Campaña M, Melendes E, Flores J, Acosta R. Administración de empresas de economía social y solidaria. MKT, Descubre; 2019.

[21] Pérez F, García R. Gestión del tiempo para estudiantes. Valencia: Facultad de Psicología, Universidad de Valencia; 2016.

[22] Flores JC. Proceso administrativo, contable en los negocios y la competitividad para la gestión económica local. MKT, Descubre; 2019.

[23] Leyva A, Arroyo J, Espejel J. Influencia de la planeación estratégica y habilidades gerenciales como factores internos de la competitividad empresarial de las Pymes. Contaduría y Administración. 2018;63:1-21.

[24] Sanchez M, Lazo V. Determinantes de la rentabilidad empresarial en el Ecuador: un análisis de corte transversal. ECA Sinergia; 2017. 